\title{
Comparative Analysis of Symptomatic and Asymptomatic SARS-CoV-2 Infection in Children
}

Jiahui Li $\underline{\mathrm{Li}},{ }^{* 1}$ MBBS, MRCPCH, M Med (Paeds), Koh Cheng Thoon, ${ }^{1}$ MBBS, MRCPCH, M Med (Paeds), Chia Yin Chong, ${ }^{1} M B B S, M$ Med, FRCPCH, Matthias Maiwald, ${ }^{2} M D$, FRCPA, Kai-qian $\underline{\mathrm{Kam}},{ }^{1}$ MBBS, MRCPCH, M Med (Paeds), Karen Nadua, ${ }^{1}$ MD, MRCPCH, M Med (Paeds), Natalie WH Tan, ${ }^{1} M B B S, M R C P C H$, Chee Fu Yung ${ }^{*},{ }^{*} 1 M B C h B, F F P H$

\begin{abstract}
Introduction: In this study, a comparison of clinical, epidemiological and laboratory parameters between symptomatic and asymptomatic children with SARS-CoV-2 infection was performed. Materials and Methods: Data from all children with laboratory confirmed SARS-CoV-2 infection admitted to KK Women's and Children's Hospital (KKH), Singapore, from January to May 2020 were analysed. Results: Of the 39 COVID-19 children included, $38.5 \%$ were asymptomatic. Household transmission accounted for $95 \%$ of cases. The presenting symptoms of symptomatic children were low-grade fever $(54.2 \%)$, rhinorrhoea $(45.8 \%)$, sore throat $(25 \%)$, diarrhoea $(12.5 \%)$ and acute olfactory dysfunction $(5.4 \%)$. Children of Chinese ethnicity (37.5\% vs $6.7 \%)$, complete blood count $(45.8 \%$ vs $6.7 \%)$ and liver enzyme abnormalities (25\% vs $7.7 \%)$ were more common in symptomatic versus asymptomatic children. All children had a mild disease course and none required oxygen supplementation or intensive care. Conclusions: The high proportion of asymptomatic infected children coupled with household transmission as the main source of paediatric COVID-19 infection underscores the importance of early screening and isolation of children upon detection of an index case of COVID-19 in a household. Symptomatic children were more likely to have abnormal laboratory parameters but they did not have a poorer outcome compared to asymptomatic cases.
\end{abstract}

Ann Acad Med Singap 2020;49:530-37

Key words: COVID-19, Paediatric, Pandemic, Singapore

\section{Introduction}

Severe acute respiratory syndrome coronavirus-2 (SARS-CoV-2) is an enveloped, single-stranded RNA virus that belongs to the betacoronavirus genus which includes 2 other RNA viruses that have caused recent important epidemics: SARS caused by SARS-CoV-1, and Middle East Respiratory Syndrome (MERS) by MERS-CoV. In late December 2019, the first cases of COVID-19 were reported in Wuhan, Hubei Province, China. ${ }^{1}$ COVID-19 rapidly spread across the globe, and the World Health Organization (WHO) declared the SARS-CoV-2 outbreak a pandemic on 11 March $2020 .^{2,3}$ Globally, there are now more than 15 million cases and over 619,000 deaths due to COVID-19. ${ }^{4}$

Singapore confirmed its first case of COVID-19 on 23 January 2020 in a Chinese national from Wuhan, and its first paediatric case on February 4, 2020. ${ }^{5,6}$ As of 24 July 2020, Singapore has reported 49,071 cases of COVID-19. ${ }^{7}$ Singapore initiated a comprehensive surveillance, testing and contact tracing strategy as

\footnotetext{
* These authors contributed equally to this article.

${ }^{1}$ Infectious Disease Service, Department of Paediatric Medicine, KK Women's and Children's Hospital, Singapore

${ }^{2}$ Department of Pathology and Laboratory Medicine, KK Women's and Children's Hospital, Singapore

Address for Correspondence: Dr Jiahui Li, Department of Paediatric Medicine, KK Women's and Children's Hospital, 100 Bukit Timah Road, Singapore 229899, Singapore.

Email: li.jiahui@singhealth.com.sg
} 
part of its public health response to contain and mitigate the spread of COVID-19. ${ }^{8}$ Close contacts were placed under compulsory quarantine for 14 days, and were assessed by telephone for fever or respiratory symptoms by public health officials during the quarantine period. Contacts who became symptomatic were transferred to a hospital for further evaluation. ${ }^{9}$ From March 2020 , as an additional measure for children, all asymptomatic paediatric close contacts of adults with COVID-19 were also screened for SARS-CoV-2 infection via nasopharyngeal (NP) sampling.

Several case series have described COVID-19 in children, the largest from China, with most describing a milder course of illness than in adults., ${ }^{2,6,10,11}$ However, the differences in characteristics between asymptomatic and symptomatic SARS-CoV-2 infection in children have not been well described. A better understanding of this differentiation will provide insight into the transmission dynamics of COVID-19, identify predictors of severity and guide clinical management of children infected with SARS-CoV-2. ${ }^{8}$ In this investigation, we compared the clinical findings, epidemiology and laboratory parameters between symptomatic and asymptomatic paediatric COVID-19 cases.

\section{Materials and Methods}

\section{Study Setting and Participants}

KK Women's and Children's Hospital (KK Hospital) is the single public specialist women's and children's hospital in Singapore with 830 beds. It is the main isolation hospital for paediatric COVID-19 cases in Singapore. We included all children with confirmed SARS-CoV-2 infection diagnosed via real-time reverse transcriptase-polymerase chain reaction (RT-PCR) from 2 January 2020 to 9 May 2020. Cases were classified as asymptomatic only if they had no clinical signs or symptoms throughout the course of their infection.

\section{Clinical Data Collection}

Demographic, clinical and laboratory data from electronic health records of children with SARS-CoV-2 infection were collected using a standardised data collection form. All cases were confirmed via RT-PCR test of nasopharyngeal (NP) samples. ${ }^{12}$ The hospital has in place a standardised protocol for the clinical management of all confirmed COVID-19 cases. Complete blood counts (CBC) and liver enzymes were performed as part of standard of care. Blood samples for SARS-CoV-2 RT-PCR were also obtained during admission. Stool and urine samples were taken for a subset of cases mainly during the early phases of the epidemic but these were subsequently discontinued. NP samples for respiratory pathogens by multiplex PCR (BioFire FilmArray RP2 Panel, Salt Lake City, UT, USA), serum samples for C-reactive protein (CRP), procalcitonin and blood cultures were collected based on clinical indication. Chest radiographs (CXR) were performed if there was a clinical suspicion of pneumonia. Children were discharged from hospital when they had negative SARS-COV-2 PCR results from NP samples on 2 consecutive days. ${ }^{13}$

The study was approved by the institutional ethics review board. Written informed consent was waived in light of the need to inform public health outbreak control policies.

\section{Data and Statistical Analysis}

SPSS statistical software (version 23; IBM, Armonk, NY, USA) was used for statistical analysis. Continuous variables were expressed as mean if normally distributed. For categoric clinical and demographic variables, differences between groups were evaluated using the chi-square test or Fisher's exact test when appropriate. The independent student t-test was applied for comparisons involving scale variables. A $P$-value of $P<0.05$ was considered statistically significant. We also performed age-stratified analysis using 3 age categories: $0-4$ years, $5-9$ years, $10-16$ years.

\section{Results}

\section{Clinical and Epidemiological Features}

39 children with laboratory confirmed SARSCoV-2 infection were admitted for isolation between 2 January 2020 and 9 May 2020. This represented approximately $70 \%$ of all paediatric cases detected in Singapore. ${ }^{14}$ Household transmission accounted for $95 \%$ of cases in our cohort (Table 1). The most common symptoms associated with COVID-19 in children were fever $(54.2 \%)$, rhinorrhoea $(45.8 \%)$, sore throat $(25 \%)$ and cough (16.7\%) (Table 1). The mean maximum recorded temperature amongst children with a fever was $38.6^{\circ} \mathrm{C}$ (range $38.0-41.0^{\circ} \mathrm{C}$ ), and the mean total duration of fever was 2.6 days (range 1-6 days). Three children $(12.5 \%)$ had diarrhoea as part of their clinical manifestation. In addition, two children (5.4\%) aged 11 and 14 years also reported loss or reduced sense of smell and taste followed by fever as their only symptoms. This lasted until day 10 of illness 
for one and continued until discharge (day 17 of illness) for the other. None of the children reported shortness of breath or chest discomfort, and none developed tachypnoea, tachycardia, hypotension or signs suggestive of Kawasaki disease during their illness.

Of the 39 paediatric cases, 19 (49\%) were asymptomatic at presentation, $18(95 \%)$ of whom were detected via testing of asymptomatic household contacts of adults with COVID-19. Subsequently, 4 of the 19 children developed symptoms during admission and hence the proportion who remained asymptomatic throughout their course of illness was $38.5 \%(15 / 39)$. The mean ages of symptomatic and asymptomatic cases were 7.8 and 8.3 years respectively $(P=0.67)$ (Table 1$)$. Similarly, there was a larger proportion of symptomatic children from $0-4$ years of age $(75 \%)$ as compared to older children $(53 \%$ in children 5-9 years and $64 \%$ in children $10-16$ years) (Table 1), but this was not statistically significant $(P=0.55)$. Children of Chinese ethnicity were most common in symptomatic COVID-19 children (37.5\%), and children of Indian ethnicity were most common

Table 1. Demographic and Clinical Features of Symptomatic and Asymptomatic Children with SARS-CoV-2 Infection

\begin{tabular}{|c|c|c|c|c|}
\hline Characteristic & Symptomatic & Asymptomatic & Total & P-value \\
\hline Number, n (\%) & $24(61.5)$ & $15(38.5)$ & 39 & \\
\hline Mean age (years) & 7.8 & 8.3 & & 0.67 \\
\hline $0-4$ years & $6(75)$ & $2(25)$ & $8(20.5)$ & 0.55 \\
\hline $5-9$ years & $9(52.9)$ & $8(47)$ & $17(43.5)$ & \\
\hline $10-16$ years & $9(64.3)$ & $5(35.7)$ & $14(35.9)$ & \\
\hline Males, n (\%) & $15(62.5)$ & $8(53.3)$ & $23(60)$ & 0.74 \\
\hline Ethnicity, n (\%) & & & & 0.08 \\
\hline Chinese & $9(37.5)$ & $1(6.7)$ & $10(25.6)$ & \\
\hline Malay & $6(25.0)$ & $5(33.3)$ & $11(28.2)$ & \\
\hline Others & $5(20.8)$ & $2(13.3)$ & $7(17.9)$ & \\
\hline Comorbidities present, n (\%) & $9(37.5)$ & $1(6.7)$ & & 0.06 \\
\hline Index is positive family member, $\mathrm{n}(\%)$ & $22(91.7)$ & $15(100)$ & $37(94.9)$ & 0.37 \\
\hline Father & $10(41.7)$ & $5(33.3)$ & $14(35.9)$ & \\
\hline Mother & $4(16.7)$ & $7(46.7)$ & $10(25.6)$ & \\
\hline Grandmother & $3(12.5)$ & $1(6.7)$ & $4(10.2)$ & \\
\hline Older sibling & $1(4.2)$ & 0 & $1(2.6)$ & \\
\hline More than 1 family member & $4(16.7)$ & $2(13.3)$ & $6(15.4)$ & \\
\hline \multicolumn{5}{|l|}{ Presenting symptoms, n (\%) } \\
\hline Blocked nose or rhinorrhoea & $11(45.8)$ & & & \\
\hline Sore throat & $6(25)$ & & & \\
\hline Cough & $4(16.7)$ & & & \\
\hline Sneezing & $3(12.5)$ & & & \\
\hline Loose stools & $3(12.5)$ & & & \\
\hline Anosmia or hyposmia & $2(5.4)$ & & & \\
\hline
\end{tabular}


amongst asymptomatic children (46.7\%), but the differences were not statistically significant $(P=0.08)$. Comorbidities were more likely to be present in symptomatic COVID-19 cases compared to asymptomatic $(37.5 \%$ vs $6.7 \%, P=0.06)$. The most common comorbidities included asthma (30\%), epilepsy (20\%), and hypothyroidism (20\%).

\section{Laboratory Parameters, Clinical Investigations and Outcomes}

Abnormalities in CBC and liver enzymes were more frequently detected on admission in symptomatic children (Table 2). On admission, 11 of 24 (45.8\%) symptomatic cases had at least an abnormal white blood cell or platelet count versus 1 of $15(6.7 \%)$ asymptomatic cases $(P=0.01)$. Neutropenia for age $(25 \%)$ followed by leukopenia for age $(12.5 \%)$ were the most common $\mathrm{CBC}$ abnormalities detected amongst symptomatic cases. The only abnormal CBC parameter detected in asymptomatic cases was neutropenia, which was detected in 1 patient. The proportion of cases with abnormal liver enzymes was similarly higher in symptomatic cases compared to asymptomatic cases $(25 \%$ versus $7.7 \%$ respectively, $P=0.22$ ). A raised alanine transaminase (ALT) level was the most likely abnormal liver enzyme especially amongst symptomatic cases (29.2\%). Both mean ALT and aspartate transaminase (AST) were higher in symptomatic children (22.6 and 31.2 U/L respectively) compared to that in asymptomatic children (15.8 and $25.6 \mathrm{U} / \mathrm{L}$ respectively) (Table 2). All haematological and liver enzyme abnormalities detected in this cohort

Table 2. Laboratory Features and Investigations of Symptomatic and Asymptomatic Children with SARS-CoV-2 Infection

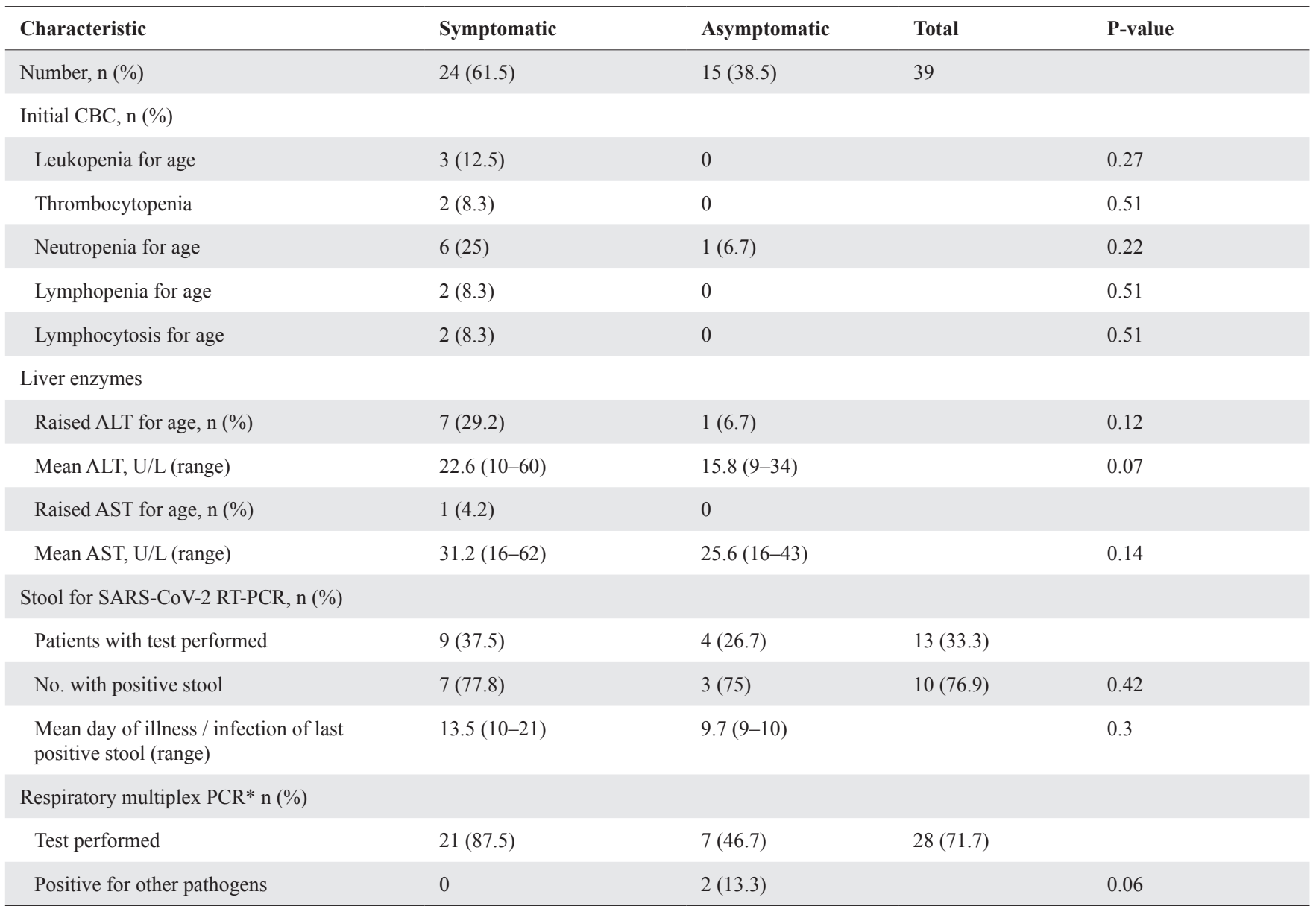

ALT: Alanine transaminase; AST: Aspartate transaminase; CBC: Complete blood count; PCR: Polymerase chain reaction; RT-PCR: Real-time polymerase chain reaction; WBC: White blood cell count.

*FilmArray RP2 Panel (BioFire, Salt Lake City, UT, USA) 
of paediatric COVID-19 cases resolved by day 29 and day 18 of illness respectively.

Thirty-five children $(89.7 \%)$ had blood tested for SARS-CoV-2 RT-PCR, of whom only 1 (2.6\%) returned positive. ${ }^{6}$ Of 39 children with SARS-CoV-2 infection, $13(33.3 \%)$ had stool tested for SARS-CoV-2 RT-PCR with a positivity rate of $76.9 \%(10 / 13)$ (Table 2). Symptomatic children also had a longer duration of viral shedding in stool compared to asymptomatic children (mean 13.5 days and 9.7 days respectively), though this was not statistically significant $(P=0.3)$ (Table 2). Six children $(15.4 \%)$ had urine tested for SARS-CoV-2 RT-PCR, and all results were negative.

Twenty-eight children (71.8\%) had NP samples screened via multiplex PCR for other respiratory pathogens (Table 2). Only 2 were positive for alternative pathogens (rhinovirus/enterovirus and respiratory syncytial virus) and both cases were asymptomatic. CRP was determined in 5 of $24(20.8 \%)$ symptomatic children for the main indication of significant temperature during admission, with a mean value of $2.4 \mathrm{mg} / \mathrm{L}$. Only one child had an abnormal CRP value of $8.2 \mathrm{mg} / \mathrm{L}$. Of the 4 children who had a CXR performed during admission, 3 were normal and 1 showed perihilar infiltrates.

All children received supportive care and symptomatic treatment, including antipyretics and antihistamines. None required oxygen supplementation or ventilatory support, and none received antiviral therapy, interferon, or corticosteroids. All 39 children were discharged well from hospital, with a mean length of stay of 15 days (standard deviation 6.5 days, range 3-30 days).

\section{Discussion}

The proportion of symptomatic and asymptomatic paediatric COVID-19 cases was $61.5 \%$ and $38.5 \%$ respectively in our cohort. We found that children of Chinese ethnicity $(37.5 \%)$ were more likely to present with COVID-19 symptoms while Indian children were more likely to be asymptomatic (46.7\%), but these differences were not statistically significant. More data and research are needed to ascertain if there are differences in response to SARS-CoV-2 due to host genetic variations. The main presenting symptoms of symptomatic children in our case series were low-grade fever $(54.2 \%)$, rhinorrhoea $(45.8 \%)$, sore throat $(25 \%)$, and diarrhoea $(12.5 \%)$. We also noted acute olfactory dysfunction (anosmia or hyposmia) and dysgeusia as presenting symptoms in $2(5.4 \%)$ children. We did not observe major differences in clinical outcome between COVID-19 children with symptoms and those without.

The proportion of asymptomatic children with laboratory-confirmed SARS-CoV-2 infection in the paediatric literature ranged from $12.9 \%-28 \%$, lower than that reported in our cohort (38.5\%). ${ }^{11,15,16}$ Our data may provide a more accurate estimate of the population asymptomatic rate in children due to the comprehensive surveillance, contact tracing and testing of exposed children regardless of symptom status as part of Singapore's public health control strategy. Although the infectivity of asymptomatic children is poorly understood, transmission from pre-symptomatic and asymptomatic adults has been described. ${ }^{17,}{ }^{18} \mathrm{We}$ noted a higher proportion of symptomatic cases in the $0-4$ years age category, in contrast to the closer proportions reported in the older age categories ( $75 \%$ symptomatic in $0-4$ years versus $52.9 \%$ in $5-9$ years and $64.3 \%$ in $10-16$ years) (Table 1 ).

Paediatric studies from China found that household transmission accounted for $70-100 \%$ of cases. ${ }^{19,20}$ Similarly, in our series, we found that family members were the main source of infection for children (95\%). The high proportion of asymptomatic children infected by household members underscores the importance of early screening and isolation of children upon detection of an index case of COVID-19 in a household..$^{21,22}$ In settings where testing capabilities may be limited, it might be useful to isolate or quarantine all paediatric household contacts regardless of presence of symptoms once a confirmed COVID-19 case is detected to curb further community transmission of SARS-CoV-2. ${ }^{23}$ The proportion of asymptomatic infected individuals in mixed adult and paediatric studies varies greatly from $1-43 \%{ }^{2,24}$ The estimated proportion of asymptomatic infections is a key parameter in epidemiological modelling studies currently used to inform public health strategy. ${ }^{25}$ There may be a need to incorporate age-stratified estimates of asymptomatic infection into modelling studies to provide more accurate estimates. ${ }^{8,26-28}$

The reported symptoms in our cohort were similar to that reported in other paediatric case series, where the most common presenting symptoms were also fever (36-41\%), cough (19-48.5\%), sore throat (36-46.2\%), and diarrhoea (8.8\%). ${ }^{11,15,29}$ However, the proportion of children reporting cough in our cohort was much lower 
$(16.7 \%)$. The reason for this is unclear but it could be the result of our limited sample size. Acute olfactory dysfunction (anosmia or hyposmia) and dysgeusia have been identified as early symptoms of SARS-CoV-2 infection in adults. ${ }^{30}$ Two children in our cohort reported these symptoms and this proportion is at the lower end of the range reported in adult COVID-19 cases $(5.1-85.6 \%){ }^{31}$ For one of our cases, hyposmia was the only presenting complaint at presentation. Therefore, it will be useful to include loss of smell or taste in screening criteria for paediatric COVID-19 cases as per adults. Presence of symptoms did not influence the outcome or treatment required, and all children in our cohort had a mild course of illness. Children have been noted to have a milder course of illness compared to adults, similar to paediatric MERS and SARS disease. ${ }^{10,32-35}$ However, recent reports of Kawasakilike disease and multisystem inflammatory syndrome in children (MIS-C) from Europe and USA described in children with asymptomatic or mildly symptomatic SARS-CoV-2 infection is worrying. ${ }^{36}$ To date, no such reports have been identified in Asia. At the time of writing, children with COVID-19 in this cohort were well at follow-up, and have not been admitted to our institution for symptoms suggestive of Kawasaki disease or MIS-C after their initial discharge.

The majority of haematological and liver enzyme abnormalities were observed amongst symptomatic children, and almost all asymptomatic children (except 1 child) had normal laboratory parameters. This suggests that infection may be localised to the nasopharynx and systemic involvement was absent in asymptomatic children. Leukopenia and neutropenia were the most common haematological abnormalities in this paediatric cohort ( $7.7 \%$ and $18 \%$ respectively). These abnormalities were consistent with that reported in other paediatric studies, and normalised on repeat testing. ${ }^{37}$ Alternative pathogens in NP samples were detected in 2 asymptomatic children. Unlike previous paediatric case series which described a more severe course of illness in children with SARS-CoV-2 and co-infections with other respiratory viruses, our patients remained asymptomatic and clinically well. ${ }^{38,39}$ More studies are required to determine how co-infections with other respiratory viruses influence severity of illness in children with COVID-19.

The poor yield of blood and urine for SARS-CoV-2 by PCR has been described previously. ${ }^{39,40}$ Of the 35 children in this case series who had blood tested for SARS-CoV-2 by RT-PCR, only one infant was positive. Viremia in the infant did not result in more severe clinical manifestation as the infant remained clinically well apart from one fever spike during the course of the illness. ${ }^{6}$ The stool SARS-CoV-2 RT-PCR positivity rate of $77 \%$ in our cohort was close to the $80-100 \%$ reported in other paediatric studies. ${ }^{19,41,42}$ In our cohort, stool positivity was detected up to 21 days of illness with longer duration noted in symptomatic cases. Persistent fecal detection of SARS-CoV-2 by RT-PCR after consecutive negative NP samples has been described, with viral detection in stool reported as late as day 33 of illness. ${ }^{19,40,43}$ Until more information is available on the viral replicability of SARS-CoV-2 isolated from stool in the late course of illness, quarantine and infection control measures should take into consideration the late shedding of SARS-CoV-2 in stool of children regardless of presence of symptoms, especially in young children and those with special needs who require caregivers to handle toileting requirements.

The major limitation of this study was its limited sample size. However, our cohort was based on a standardised national public health surveillance and comprehensive contact tracing strategy to actively identify cases, and represented $70 \%$ of all paediatric cases detected in Singapore. SARS-CoV-2 testing was performed using pre-determined case definitions for suspect cases. ${ }^{9}$ Furthermore, our protocol to proactively test all paediatric household contacts independent of symptom status provided a more accurate rate for asymptomatic cases in children. We also utilised a standardised clinical management and basic blood parameter testing protocol for all our admitted cases.

\section{Conclusion}

Household transmission remains a major mode of transmission of SARS-CoV-2 infection in the paediatric population. A significant proportion $(38.5 \%)$ of paediatric patients with SARS-CoV-2 infection remain asymptomatic, with minimal evidence of systemic involvement. The high proportion of asymptomatic infected children coupled with household transmission as the main source of paediatric COVID-19 infection underscores the importance of early screening and isolation of children upon detection of an index case of COVID-19 in a household. Although symptomatic children were more likely to have abnormal laboratory parameters, they did not have a poorer outcome compared to asymptomatic children, and all children in our cohort recovered from SARS-CoV-2 infection. 


\section{REFERENCES}

1. Zhu N, Zhang D, Wang W, Li X, Yang B, Song J, et al. Novel coronavirus from patients with pneumonia in China, 2019. N Engl J Med 2020;382:727-733.

2. Wu Z, McGoogan JM. Characteristics of and important lessons from the coronavirus disease 2019 (COVID-19) outbreak in China: summary of a report of 72314 cases from the Chinese Center for Disease Control and Prevention. JAMA 2020 Feb 24; Online ahead of print.

3. World Health Organization rolling updates on coronavirus disease (COVID-19). World Health Organization. https://www.who.int/ emergencies/diseases/novel-coronavirus-2019/events-as-they-happen. Accessed on 24 July 2020.

4. World Health Organization coronavirus disease (COVID-2019) situation reports. World Health Organization. https://www.who.int/ emergencies/diseases/novel-coronavirus-2019/situation-reports/. Accessed on 24 July 2020.

5. Confirmed imported case of novel coronavius infection in Singapore. Ministry ofHealth, Singapore. https://www.moh.gov.sg/news-highlights/ details/confirmed-imported-case-of-novel-coronavirus-infection-insingapore-multi-ministry-taskforce-ramps-up-precautionary-measures. Accessed on 24 July 2020

6. Kam KQ, Yung CF, Cui L, Lin RTP, Mak TM, Maiwald M, et al. A well infant with Coronavirus disease 2019 (COVID-19) with high viral load. Clin Infect Dis 2020;71:847-849.

7. Updates on COVID-19 (Coronavirus Disease 2019) local situation. https://www.moh.gov.sg/covid-19. Accessed on 24 July 2020.

8. Hsu LY, Chia PY, Lim JF. The novel coronavirus (SARS-CoV-2) epidemic. Ann Acad Med Singapore 2020 Mar 16;49:105-107.

9. Ng Y, Li Z, Chua YX, Chaw WL, Zhao Z, Er B, et al. Evaluation of the effectiveness of surveillance and containment measures for the first 100 patients with COVID-19 in Singapore - January 2-February 29, 2020. MMWR Morb Mortal Wkly Rep 2020;69:307-311.

10. Ludvigsson, JF. Systematic review of COVID-19 in children shows milder cases and a better prognosis than adults. Acta Paediatr 2020;109:1088-1095.

11. Qiu H, Wu J, Hong L, Luo Y, Song Q, Chen D. Clinical and epidemiological features of 36 children with coronavirus disease 2019 (COVID-19) in Zhejiang, China: an observational cohort study. Lancet Infect Dis 2020; 20:689-696.

12. Corman VM, Landt O, Kaiser M, Molenkamp R, Meijer A, Chu DKW et al. Detection of 2019 novel coronavirus (2019-nCoV) by real-time RT-PCR. Euro Surveill 2020;25:2000045.

13. Wong JEL, Leo YS, Tan CC. COVID-19 in Singapore-current experience: critical global issues that require attention and action. JAMA 2020 Feb 20; Online ahead of print.

14. No severe inflammatory symptoms in infected children in Singapore. The Straits Times. https://www.straitstimes.com/singapore/ no-severe-inflammatory-symptoms-in-infected-children-heremoh?xtor $=$ CS3-18\&utm source $=$ STiPhone\&utm medium $=$ share\&utm term $=2020-05-29 \% 209 \% 3$ A05\%3A51. Accessed on 24 July 2020.

15. Lu X, Zhang L, Du H, Zhang J, Li YY, Qu J, et al. SARS-CoV-2 infection in children. N Engl J Med 2020;382:1663-1665.

16. Dong Y, Mo X, Hu Y, Qin X, Jiang F, Jiang Z, et al. Epidemiology of COVID-19 among children in China. Pediatrics 2020;145:e20200702.

17. Arons MM, Hatfield KM, Reddy SC, Kimball A, James A, Jacobs JR, et al. Presymptomatic SARS-CoV-2 infections and transmission in a skilled nursing facility. N Engl J Med 2020;382:2081-2090.
18. Wei WE, Li Z, Chiew CJ. Presymptomatic transmission of SARS-CoV-2 - Singapore, January 23-March 16, 2020. MMWR Morb Mortal Wkly Rep 2020;69:411-415.

19. Cai J, Xu J, Lin D, Yang Z, Qu Z, Zhang Y, et al. A case series of children with 2019 novel coronavirus infection: clinical and epidemiological features. Clin Infect Dis 2020 Feb 20; Online ahead of print.

20. Su L, Ma X, Yu H, Zhang Z, Bian P, Han Y, et al. The different clinical characteristics of corona virus disease cases between children and their families in China - the character of children with COVID-19. Emerg Microbes Infect 2020;9:707-713.

21. Yung CF, Kam KQ, Chong CY, Nadua KN, Li JH, Tan NWH, et al. Household transmission of SARS-CoV-2 from adults to children. J Pediatr 2020;S0022-3476(20)30852-0.

22. Lim TK. The Facts, Fallacies and Uncertainties about Coronavirus Disease 2019 (COVID-19). Ann Acad Med Singapore 2020;49:343-5.

23. Viner RM, Russell SJ, Croker H, Packer J, Ward J, Stansfield C, et al. School closure and management practices during coronavirus outbreaks including COVID-19: a rapid systematic review. Lancet Child Adolesc Health 2020;4:397-404.

24. Gudbjartsson DF, Helgason A, Jonsson H, Magnusson OT, Melsted P, Gudmundur LN, et al. Spread of SARS-CoV-2 in the Icelandic population. N Engl J Med 2020;382:2302-2315.

25. Koo JR, Cook AR, Park M, Sun Y, Sun H, Lim JT, et al. Interventions to mitigate early spread of SARS-CoV-2 in Singapore: a modelling study. Lancet Infect Dis 2020;20:678-688.

26. Ferguson NM, Laydon D, Nedjati-Gilani G, Imai N, Ainslie K, Baguelin $\mathrm{M}$ et al. Report 9: Impact of non-pharmaceutical interventions (NPIs) to reduce COVID-19 mortality and healthcare demand. 16 March 2020. https://www.imperial.ac.uk/media/imperial-college/ medicine/sph/ide/gida-fellowships/Imperial-College-COVID19-NPImodelling-16-03-2020.pdf. Accessed on 24 July 2020.

27. Park SW, Cornforth DM, Dushoff J. The time scale of asymptomatic transmission affects estimates of epidemic potential in the COVID-19 outbreak. Epidemics 2020;31:100392.

28. Adam D. Special report: the simulations driving the world's response to COVID-19. Nature 2020;580:316-318.

29. de Souza TH, Nadal JA, Nogueira RJN, Pereira RM, Brandão MB. Clinical manifestations of children with COVID-19: A systematic review. Pediatr Pulmonol 2020;55:1892-1899.

30. Giacomelli A, Pezzati L, Conti F, Bernacchia D, Siano M, Oreni L, et al. Self-reported olfactory and taste disorders in SARS-CoV-2 patients: a cross-sectional study. Clin Infect Dis 2020;71:889-890.

31. Lechien JR, Chiesa-Estomba CM, De Siati DR, Horoi M, Le Bon $\mathrm{SD}$, Rodriguez A, et al. Olfactory and gustatory dysfunctions as a clinical presentation of mild-to-moderate forms of the coronavirus disease (COVID-19): a multicenter European study. Eur Arch Otorhinolaryngol 2020;277:2251-2261.

32. Thabet F, Chehab M, Bafaqih H. Middle East respiratory syndrome coronavirus in children. Saudi Med J 2015;36:484-486.

33. Leong HN, Earnest A, Lim HH, Chin CF, Tan C, Puhaindran ME, et al. SARS in Singapore--predictors of disease severity. Ann Acad Med Singapore 2006;35:326-31.

34. Stockman LJ, Massoudi MS, Helfand R, Erdman D, Siwek AM, Anderson LJ, et al. Severe acute respiratory syndrome in children. Pediatr Infect Dis J 2007;26:68-74. 
35. Puthucheary J, Lim D, Chan I, Chay OM, Choo P. Severe acute respiratory syndrome in Singapore. Arch Dis Child 2004;89:551-556.

36. Belhadjer Z, Méot M, Bajolle F, Kraiche D, Legendre A, Abakka S, et al. Acute heart failure in multisystem inflammatory syndrome in children (MIS-C) in the context of global SARS-CoV-2 pandemic. Circulation 2020;142:429-436.

37. Henry BM, Lippi G, Plebani M. Laboratory abnormalities in children with novel coronavirus disease 2019. Clin Chem Lab Med 2020; 58:1135-1138

38. Sun D, Li H, Lu XX, Xian H, Ren J, Zhang FR, et al. Clinical features of severe pediatric patients with coronavirus disease 2019 in Wuhan: a single center's observational study. World J Pediatr 2020;16:251-259.

39. Tagarro A, Epalza C, Santos M, Sanz-Santaeufemia FJ, Otheo E, Moraleda C, et al. Screening and severity of coronavirus disease
2019 (COVID-19) in children in Madrid, Spain. JAMA Pediatr 2020; e201346; Online ahead of print.

40. Wölfel R, Corman VM, Guggemos W, Seilmaier M, Zange S, Müller MA, Niemeyer D, et al. Virological assessment of hospitalized patients with COVID-2019. Nature 2020;581:465-469.

41. Zhang T, Cui X, Zhao X, Wang J, Zheng J, Zheng G, et al. Detectable SARS-CoV-2 viral RNA in feces of three children during recovery period of COVID-19 pneumonia. J Med Virol 2020;92:909-914

42. Xu Y, Li X, Zhu B, Liang H, Fang C, Gong Y, et al. Characteristics of pediatric SARS-CoV-2 infection and potential evidence for persistent fecal viral shedding. Nat Med 2020;26:502-505.

43. Xing YH, Ni W, Wu Q, Li WJ, Li GJ, Wang WD, et al. Prolonged viral shedding in feces of pediatric patients with coronavirus disease 2019. J Microbiol Immunol Infect 2020;53:473-480. 